\title{
ROLE OF THE NITRIC OXIDE (NO) IN THE REGULATION OF STEROIDOGENESIS IN PLACENTA DURING PHYSIOLOGICAL PREGNANCY AND PREECLAMPSIA (EXPERIMENTAL STUDY)
}

\author{
*TAMAR V. SANIKIDZE ${ }^{1}$, LEVAN A. CHEISHVILI ${ }^{1}$, NANA V. KIPIANI ${ }^{1}$, EKA R. SHEKILADZE ${ }^{1,2}$, \\ NINA V. KIPIANI ${ }^{1}$, GUBAZ Z. SHARASHENIDZE ${ }^{1}$, EDUARD N. CHIKVAIDZE ${ }^{2}$ \\ ${ }^{1}$ Tbilisi State Medical University, 33, Vazha Pshavela Ave, 0186, Tbilisi, Georgia, ${ }^{2}$ Javakhishvili Tbilisi State University, 1, Chavchavadze Ave., \\ 0179, Tbilisi, Georgia \\ *Corresponding author: Tamar V. Sanikidze Email: tsanikidze@tsmu.edu
}

Received July 08, 2019; accepted September 12, 2019; published online October 02, 2019

\begin{abstract}
The aim of the study was to establish the role of nitric oxide (NO) in the regulation of steroidogenesis in the placenta during physiological pregnancy and experimental preeclampsia (PE) in rats. EPR centers of the placenta, free NO and its metabolites were determined by the Electron Paramagnetic Resonance (EPR) method. At the last stage of pregnancy in the EPR spectra of the rats' placenta with PE alterations of the signals intensity of mitochondrial steroidogenic electron transport proteins were detected: the FeS-centers of adrenodoxin decreased, the ferricytochrome P-450 increased, the free NO content decreased, and the complexes of NO with heme (HbNO) and non-heme iron (FeSNO) were detected. These data indicate the violation of placental steroidogenesis, which is confirmed by a decrease in the level of progesterone in blood. Therefore, the nitrosylation of mitochondrial proteins is an important redox-dependent mechanism of regulation of the intensity of steroidogenesis.
\end{abstract}

\section{INTRODUCTION}

Preeclampsia (PE) is observed in $4-10 \%$ of pregnant women. It is clinically manifested during the second part of pregnancy as high blood pressure, proteinuria, and edema, and its early diagnosis is a big problem (North et al, 2011; Walker, 2000; Redman et al, 2005; Manandhar et al, 2013; George et al, 2012). An important pathogenic link of PE is hypoxia of the uteroplacental tissues, accompanied by damage to the vascular endothelium and the release of number of mediators that violate vascular tonus and microcirculation in the maternal organism (Crews et al, 2000; Wu et al, 2016; Wang et al, 2014; Roberts et al, 2001). In most theories, it is assumed that the pathogenesis of $\mathrm{PE}$ is associated with impaired remodeling of the spiral artery and insufficient trophoblastic invasion. The placenta plays a central role in PE, as evidenced by the rapid disappearance of disease symptoms after delivery or elective removal of the placenta, but not fetus (Chan et al, 1975; Dash et al, 2003). We hypothesized that a local chronic decrease in the perfusion of the placenta/uterus can cause metabolic disorders typical of PE.

The important role in the regulation the functions of the placenta belongs to nitric oxide (NO), redoxsensitive retrograde messenger which is actively involved in the regulation of placental metabolism, differentiation and implantation of the blastocyst (Dash et al, 2003), mobility, invasion and apoptosis of trophoblasts (Sladek et al, 1997; Kingdom et al, 1997), uteroplacental perfusion.

One of the main functions of the human placenta is to produce the steroid hormone progesterone, which is essential for maintaining pregnancy. Progesterone plays a multifactorial role in the regulation of homeostasis during pregnancy - contributes to maintaining electrolyte balance, regulation of basal vascular tone, cellular and humoral immunity, production of cytokines and antibodies against autoantigens (Klimek et al, 1998; Wan et al, 2018). During physiological pregnancy, the level of progesterone in the blood usually rises (about 510 times higher than the peak level of progesterone observed during the menstrual cycle). In women with preeclampsia, a decrease in the level of this hormone compared with the control level was found (Granger et $a l$, 2006), which may be associated with impaired placental homeostasis. The mechanisms involved in the regulation of placental steroidogenesis are not fully understood. To establish the role of the hypoxic placenta in the pathogenesis of PE, we investigated the regulatory mechanisms of steroidogenesis and 
metabolism of free nitric oxide (NO) in the placenta during physiological pregnancy and pregnancy complicated by experimental PE in rats, induced by a chronic reduction in the uteroplacental perfusion (CRUPP).

\section{MATERIAL AND METHOD}

\section{Experimental studies.}

Studies were carried out on 6-7month old Wistar female rats (Sengupta et al, 2013). Animals were kept in standard cages under standard laboratory conditions at a temperature of $25 \pm 2{ }^{\circ} \mathrm{C}$, a relative humidity $(50 \pm 15 \%)$, pregnancy conventional microbiological conditions and normal circadian rhythm (12-h dark/12-h light) and fed with normal diet and water ad libitum. All experimental procedures were performed following Guidelines for Use and Care of Animals; the Academic council of the Tbilisi State Medical University (Tbilisi, Georgia) approved the study protocol.

Intact female rats were kept separately (to prevent their olfactory stimulation by male pheromones and extending the diestrus) during 2 weeks; after they were placed with males ( 3 females per male) and after 4 days (the estrous cycle in most female rats is resumed every 3 days) were examined for vaginal plug (which confirms pregnancy). Pregnant rats (pregnancy was confirmed in $95 \%$ of the experimental female rats) were separated from the males (5 rats per cage). The control group (group 1, 40 rats) was represented by intact nonpregnant rats (group 1i, 10 rats) and pregnant rats with physiological pregnancy - non-operated (group 1a, 15 rats) and sham-operated (group $1 \mathrm{~b}, 15$ rats) rats. To simulate PE (group 2, 15 rats), the animal model of the CRU-PP was used: uteroplacental perfusion pressure was mechanically reduced according to the modified model of Alexander et al., which leads to hypertension and proteinuria in pregnancy narrowed aortic lumen, by one-third of its diameter $(0.2 \mathrm{~mm})$. Surgery was made at the end of the first week (the 8th day) of gestation and then the abdomen was closed tightly. In experimental rats at the end of 2nd (15th day) and 3rd (21th day) week of gestation (the duration of pregnancy in rats is 21-23 days) blood samples were taken from the tail vein for the examination of the progesterone and free NO content. At the end (on the 21st day) of the pregnancy, the pregnant animals (groups 1a, 1b and 2) were sacrificed by decapitation and the placenta was removed for further investigation by the EPR method. All rats (groups 1a, 1b, 2) that underwent surgical procedures and decapitation were anesthetized with $2 \%$ ether.

Electron Paramagnetic Resonance (EPR) spectroscopic studies. We used the Electron Paramagnetic Resonance (EPR) spectroscopy as the only technique that has the potential for the direct detection of free radicals in biological systems (Rice-Evans, Diplock,1992; Nonaka et al, 1989; Kozlov et al, 2003; Kirima et al, 2003). Paramagnetic centers in the EPR spectrum was identified by the g-factor (a parameter characterizing each paramagnetic center (EPR signal) ( $g$ $=h v / \beta H)$ ) corresponding to the structure of the center and symmetry of the surrounding chemical groups. For direct detection of free radicals in tissues, lowtemperature EPR measurements were performed using an EPR spectrometer RE-1307 (X-band, Chernogolovka, Russia). Quickly removed samples of the placenta (5 animals from each (1a, 1b and 2) group) were minced with surgical scissors (each sample $\sim 0.5 \mathrm{~g}$ ), placed in plastic tubes and then frozen in liquid nitrogen. EPR signals of the placenta samples were measured at liquid nitrogen temperature $(T=77 \mathrm{~K})$ in quartz dewar (modulation frequency - $100 \mathrm{kHz}$, microwave power - $20 \mathrm{~mW}$, amplitude of the second modulation - 0,1 mT, field scan - $10 \mathrm{mT}$, sweep time $200 \mathrm{~s}$, time constant - $80 \mathrm{~ms}$ ). The magnetic field was calibrated with a standard sample of $\mathrm{Mn}^{2+}$-doped $\mathrm{MgO}$ (calibrated by proton magnetic resonance magnetometer at the given microwave frequency), giving a sextet of paramagnetic centers with $g$ values of $(2.14,2.08,2.03$, $1.98,1.93$, and 1.86).

The EPR signals of cytochrome P-450 $\left(\mathrm{g}_{\mathrm{y}}=1.92, \mathrm{~g}_{\mathrm{x}}=\right.$ $2.25, \mathrm{~g}_{\mathrm{z}}=2.42$ ). iron-sulfur $(\mathrm{FeS})$ containing centers of adrenodoxin $\left(g_{\|}=1.94, g_{\perp}=2.02\right)$, nitrosyl complexes of heme $(\mathrm{HbNO})\left(\mathrm{g}_{\mathrm{c}}=2.01\right)$, and nonheme iron (FeSNO) $(\mathrm{g}=2.03)$ were identified by their g-factors (Simpson \& Miller, 1978; Pulatova et al., 1989; Tuckey et al., 2001) (calculated according to the signal's position relative to the spectral lines of the standard sample $\left(\mathrm{Mn}_{2}\right.$ in $\mathrm{MgO}$ powder)) (Kirima et al, 2003; Gainutdinov et al, 2009).

In separate subgroups of the animal spin-trap Sodium Diethyldithiocarbamate (DETC) (Sigma Aldri ch, St Louis, MO, USA) was used to record free NO in blood of intact and pregnant rats on the 15 th and $21 \mathrm{st}$-day of gestation. DETC solution $(500 \mathrm{mg} / \mathrm{kg})$ and FeSO4 (50 $\mathrm{mg} / \mathrm{kg})+$ sodium citrate $(37 \mathrm{mg} / \mathrm{kg})$ in separate injections was administrated intraperitoneally to 5 nonpregnant rats from group $1 \mathrm{i}$ and 5 pregnant animals from each group $1 \mathrm{a}, 1 \mathrm{~b}$ and 2 on the 15 th, and 5 pregnant animals from each group $1 \mathrm{a}, 1 \mathrm{~b}$ and 2 on the 21 st day of pregnancy) immediately after preparation. After 10 minutes of the injection (Galagan et al, 1997; Sanikidze et al, 2012), the blood taken from the tail vein was placed in plastic tubes and stored in liquid nitrogen until the examination of free NO content. The EPR spectra of $\mathrm{NO}-\mathrm{Fe}^{2+}(\mathrm{DETC})_{2}$ complexes were measured at a temperature $77 \mathrm{~K}$ (measurement conditions are the same as above) (Galagan et al, 1997; Sanikidze et al, 2012). 
The peak-to-peak amplitudes of the EPR signals of the heme iron complexes of cytochrome P-450 - amplitude of peak with $\mathrm{g}_{\mathrm{x}}=2.25$, non-heme iron-sulfur $(\mathrm{FeS})$ containing centers of adrenodoxin - peak $\mathrm{g}_{\|}=1.938$, nitrosyl complexes of heme iron (HbNO) - amplitude (a) of the 2nd hyperfine components of the $\mathrm{g}=2.012$, $\mathrm{A}^{\mathrm{N}}=1.7 \mathrm{mT}$ (Jakubowska et al, 2013; Dutka et al, 2019) of the triplet signal, nonheme iron complexes $($ FeSNO) - peak with $\mathrm{g}=2.03$ and spin-trapped $\mathrm{NOFe}^{2+}(\mathrm{DETC})_{2}$ complexes - amplitude (a) of the third (high-field) hyperfine component of the signal $\mathrm{g}=$ 2,038 (Plonka et al, 2003), were used to expression of the EPR signals intensity. The intensity $\left(\mathrm{I}_{\mathrm{A}}\right)$ of the EPR signals was determined in arbitrary units $(\mathrm{mm} / \mathrm{mg})$ - the ratio of peak's amplitude ( $\mathrm{mm}$ ) to the mass of tissue sample (mg). To standardize the measurement conditions of the sample, the ratio of the intensities of the EPR signals of the sample of the placenta and the 2nd component of the standard sample $\left(\mathrm{Mn}_{2}\right.$ ions in the $\mathrm{MgO}$ powder) was determined (Sanikidze \& Chikvaidze, 2016).

The free NO content in blood was calculated from the calibration curve for the amplitude of the third hyperfine component of the EPR signal of $\mathrm{NOFe}^{2+}(\mathrm{DETC})_{2}$, prepared at various concentrations $(1-20 \mu \mathrm{M})$ of the NO-donor methylamine hexamethylene methylamine dinitric oxide (MAHMA NONOate) (Fink et al, 2006; Beridze et al, 2011).

The peak-to-peak amplitude of the 2nd hyperfine components of the $\mathrm{g}=2.012, \mathrm{~A}^{\mathrm{N}}=1.7 \mathrm{mT}$ triplet signal was used to quantitatively compare the signals detected in the placenta. The amount of detected NO was determined from the calibration curve for intensity of the ESR signal of rats erythrocytes treated with known concentrations of nitrite $(1-25 \mu \mathrm{M})$ and $\mathrm{Na}_{2} \mathrm{~S}_{2} \mathrm{O}_{4}(20$ mM) (Kozliv et al, 1996; Plonka, et al, 1999, Lobysheva et al., 2013).

Progesterone content in the blood. In experimental rats at the end of 2 nd (15th day) and 3rd (21th day) of gestation weeks (5 animals in each group in each semester), blood samples were taken from the tail vein for the examination of progesterone content. Total progesterone content in the rats' blood was determined using the ELISA kit for testing rats' progesterone (Enzo Life Science).

Blood pressure measurement. Blood pressure (systolic and diastolic) was determined in all pregnant rats on day 19 of gestation; pressure was measured on the tail vein with the use of specific cuff by equipment „Systola“ (Neurobotics, Zelenograd, Russia) and oscillograph. Mean arterial pressure (MAP) was calculated according to the formula:

$\mathrm{MAP}=\mathrm{P}_{\text {diastolic }}+\left(\mathrm{P}_{\text {systolic }}-\mathrm{P}_{\text {diastolic }}\right) / 3$

Statistical analysis. Statistical analysis of the data was carried out using the SPSS-11 for Windows. Data were reported as mean $\pm \mathrm{SD}$. For normally distributed variables, the Student $t$-test was applied.

\section{RESULTS}

\section{EPR spectra of rats' placenta}

In the rats' placenta (groups 1a, 1b, 2), at the end of the 3rd week of pregnancy, the EPR signals of heme iron complexes of cytochrome P-450 $\left(\mathrm{g}_{\mathrm{y}}=1.92, \mathrm{~g}_{\mathrm{x}}=\right.$ 2.42, $\left.\mathrm{g}_{\mathrm{z}}=2.25\right)$ and non-heme iron-containing FeS centers of adrenodoxin $\left(\mathrm{g}_{\|}=1.941, \mathrm{~g}_{\perp}=2.02\right)$ (Simpson \& Miller, 1978; Pulatova et al., 1989; Tuckey et al., 2001) (Fig. 1) were measured. These EPR signals are signals of the electron carriers of the steroidogenic mitochondrial chain of the placenta. In the EPR spectra of the placenta of the sham-operated rats (group 1b) the intensity of EPR signals of adrenodoxin FeS-centers and cytochrome P-450 was similar to group la. In the EPR spectra of placenta of the pregnant rats with an experimenttal model of HCU-PP (group 2) the intensity of EPR signal of adrenodoxin FeS-centers decreased (by $60 \%$ ), the intensity of EPR signal of cytochrome P-450 increased (by 130\%) compared to the control level (Fig. 2). In the EPR spectra of the rats' placenta with experimental CRU-PP in the third week of gestation signals of non-heme iron (FeSNO) $(g=2.03)$ (Fig. 1) and nitrosyl complexes of heme $(\mathrm{HbNO})\left(g_{c}=2.012\right)$ (Fig. 3) were detected; these signals were not detected in the placenta of control animals (group 1a, 1b). 

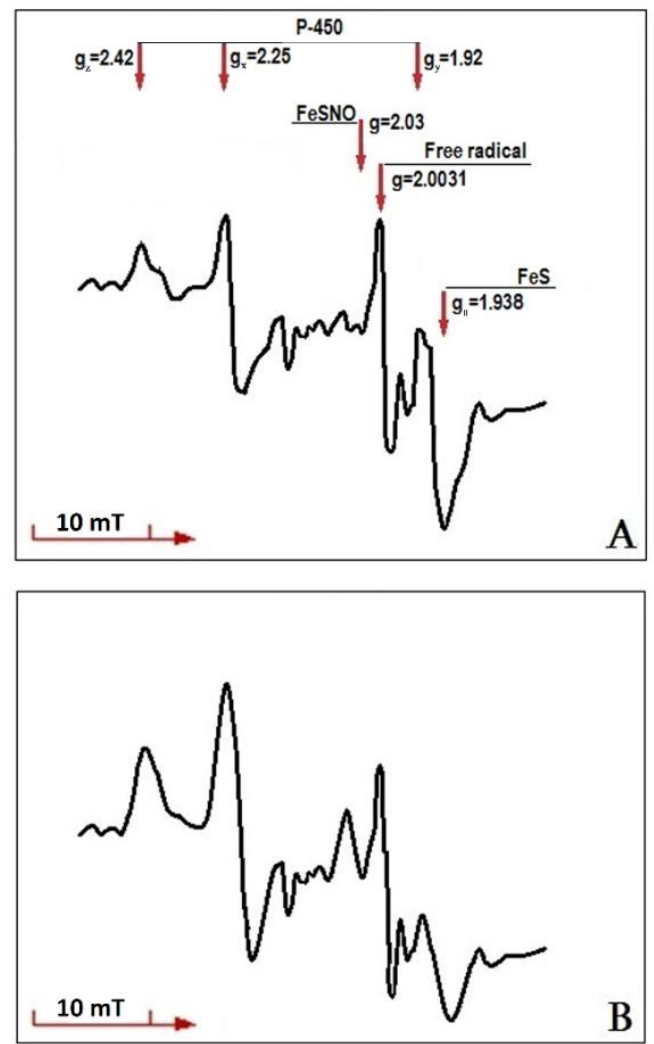

Fig. 1. EPR spectra of rats' placenta on the 3rd week of gestation (A - control group 1a; B - animal model of CRU-PP, group 2) (modulation frequency - $100 \mathrm{kHz}$, microwave power - $20 \mathrm{~mW}$, amplitude of second modulation - 0,1 mT, field scan - $10 \mathrm{mT}$, sweep time - $200 \mathrm{~s}$, time constant $-80 \mathrm{~ms}, \mathrm{~T}=77 \mathrm{~K})$.

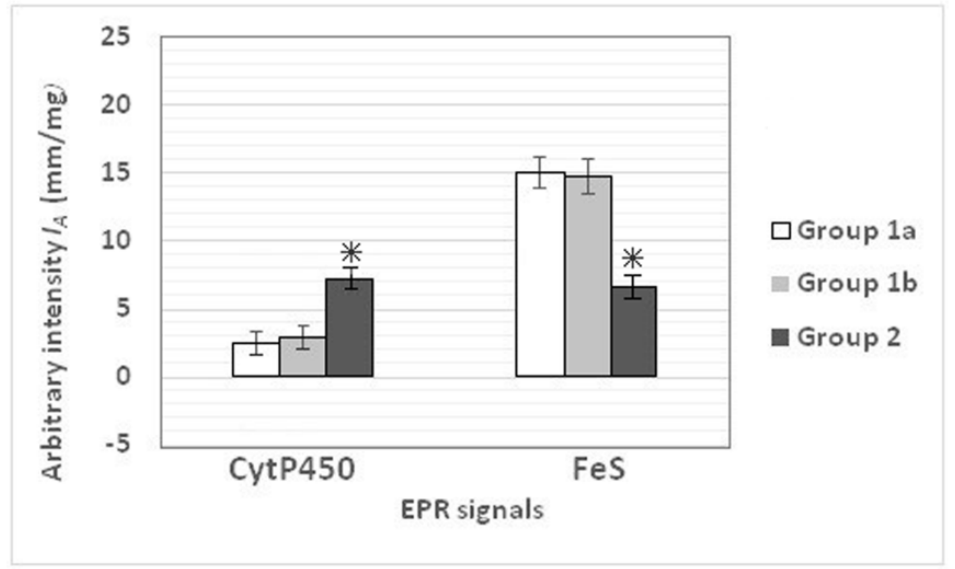

Fig. 2. The EPR signals intensity of hemic (CytP450) and non-hemic iron (adrenodoxin FeS-centres) containing complexes in rats' placenta during physiological and complicated by CRU-PP pregnancy (group 1a - control non-operated rats (5 animals); group 1b - control shamoperated rats (5 animals); group 2 - operated rats with CRU-PP (5 animals)) (Bars marked with an asterisk are a significant difference in the control values; $\mathrm{P} \leq 0.05$, according to Student's t-test (Mean $\mp \mathrm{SD})$ ). 


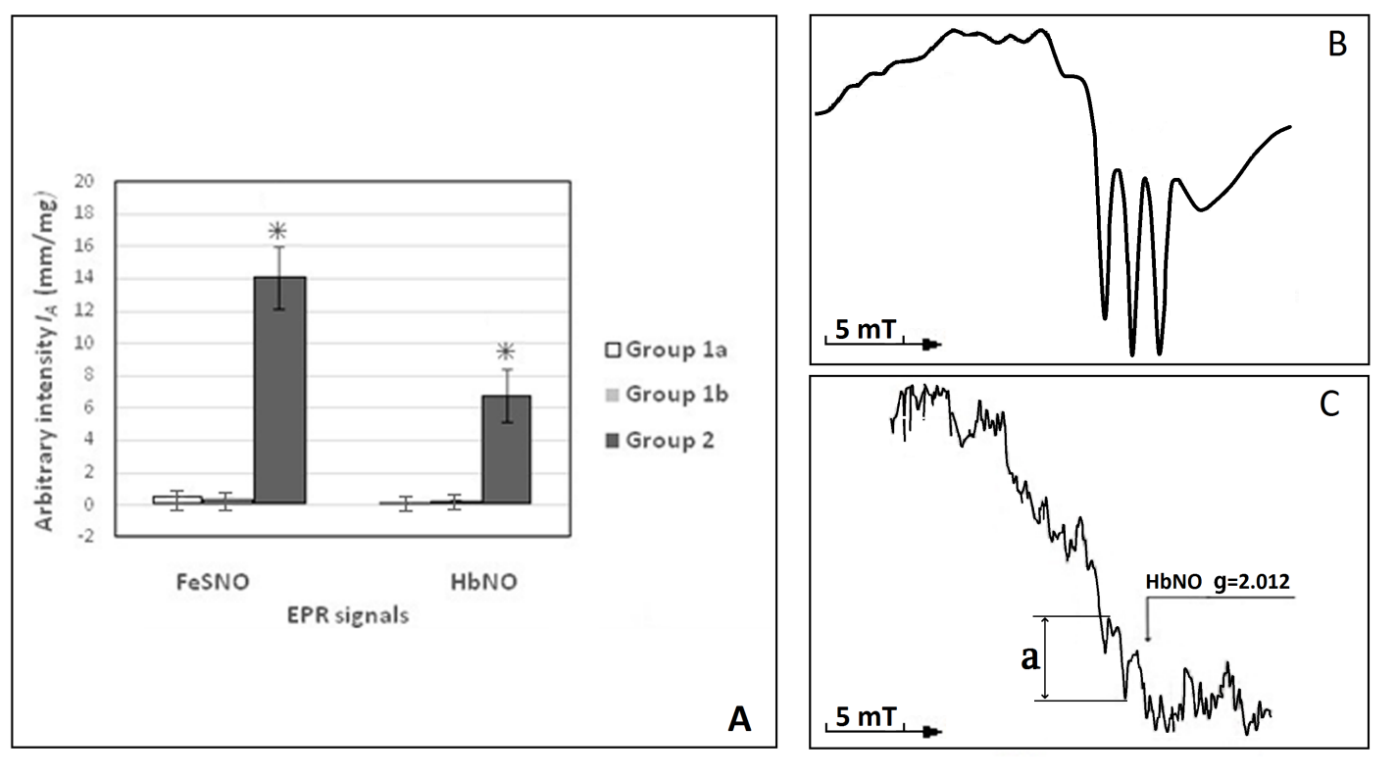

Fig. 3. A - The EPR signals intensity of complexes of nitrosylated non-heme $(\mathrm{FeSNO})(\mathrm{g}=2.03)$ and heme iron $(\mathrm{HbNO})\left(\mathrm{g}_{\mathrm{c}}=2.012\right)$ in the placenta of pregnant rats with experimental CRU-PP (group 1a - control non-operated rats (5 animals); group 1b - control sham-operated rats (5 animals); group 2 - operated rats with CRU-PP (5 animals)) (Bars marked with an asterisk are a significant difference in the control values; $\mathrm{P} \leq 0.05$, according to Student's t-test (ҒSD)). B - The "triplet signal" on the EPR spectrum of chemically pure nitrosyl hemoglobin, exposed to the increasing NO concentration, in the absence of allosteric agents, anaerobic conditions (77K) (Dutka et al., 2019). C - Typical EPR signal of hemic iron nitrosyl complexes (HbNO) $\left(\mathrm{g}_{\mathrm{c}}=2.012\right)$ in the placenta of pregnant rats with reduced uterine perfusion at the end of week III. (High frequency (modulation frequency - $100 \mathrm{kHz}$, microwave power - $20 \mathrm{~mW}$, amplitude of second modulation - 0,1 mT, field scan - $10 \mathrm{mT}$, sweep time - $200 \mathrm{~s}$, time constant - $80 \mathrm{~ms}, \mathrm{~T}=77 \mathrm{~K}$ ).
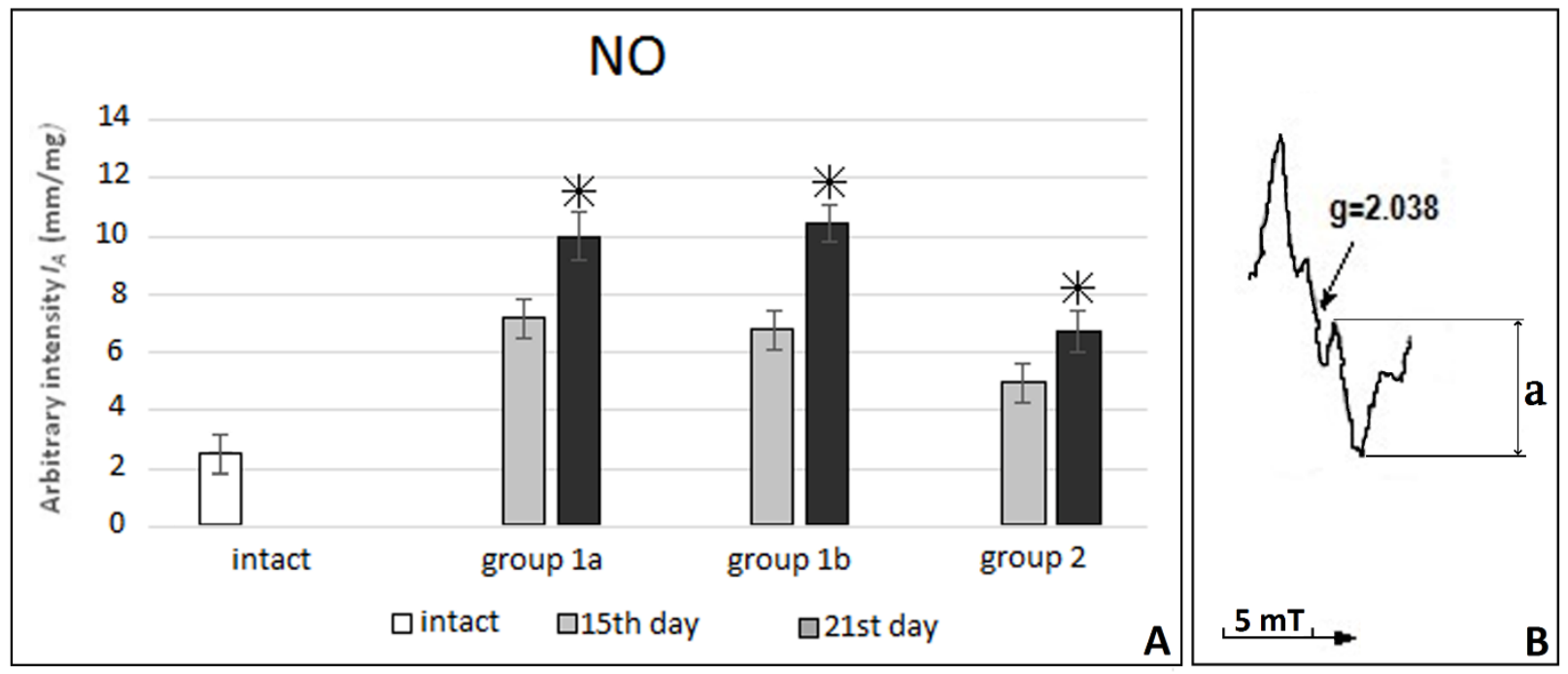

Fig. 4. A - The intensity of free nitric oxide's (NO) EPR signal in rats' placenta during physiological and complicated by CRU-PP pregnancy (group $1 \mathrm{i}$ - intact nonpregnant rats ( 5 animals); $1 \mathrm{a}$ - control non-operated rats (5 animals); group $1 \mathrm{~b}$ - control sham-operated rats (5 animals); group 2 - operated rats with CRU-PP (5 animals)). (Bars marked with an asterisk - a significant difference between the values in the second and third trimester; $\mathrm{P} \leq 0.05$, according to Student's t-test (Mean $\mp$ SD). B - Typical EPR signal of spin-trapped free $\mathrm{NO}\left(\mathrm{g}_{\mathrm{c}}=2.038\right)$ in the rats blood (modulation frequency - $100 \mathrm{kHz}$, microwave power - $20 \mathrm{~mW}$, amplitude of second modulation - 0,1 $\mathrm{mT}$, field scan - $10 \mathrm{mT}$, sweep time - $200 \mathrm{~s}$, time constant - $80 \mathrm{~ms}, \mathrm{~T}=77 \mathrm{~K})$. 
EPR spectra of rats' blood. The intensity of the EPR signal of the spin-trapped NO in the blood of control and sham-operated rats (group 1a, 1b) increased with an increase in the gestation period (on day 21 compared with 15th day increased by $42-57 \%$, correspondingly ( $\mathrm{P}$ $\leq 0.05)$ ) and on the end of the 3rd week was 4 times higher than the NO content in the blood of non-pregnant intact rats (group 1i). In the blood of rats with experimental CRU-PP (group 2), the level of the spintrapped NO was relatively low and by the end of pregnancy (day 21st) was $68 \%$ of control level $(\mathrm{P} \leq$ 0.05) (Fig. 4).
Progesterone content in the blood of pregnant rats. Fig. 5 shows the results of the study of the total progesterone content in the blood of rats during physiological (group 1a, 1b) and complicated with PE (group 2) pregnancy is shown. As follows from Fig. 5, the level of progesterone in the blood increased with an increase in the gestation age (by 3rd week increased by $41 \%$ compared with 2 nd week $(\mathrm{P} \leq 0.05))$ in all groups of experimental animals, but its level in animals with a physiological pregnancy (control and sham-operated animals (groups 1a, 1b)) exceeds the level of progesterone in the blood of animals with an experimental CRU-PP (2nd week - by $33 \%(\mathrm{P} \leq 0.05)$, 3rd week - by $50 \%(\mathrm{P} \leq 0.05))$.

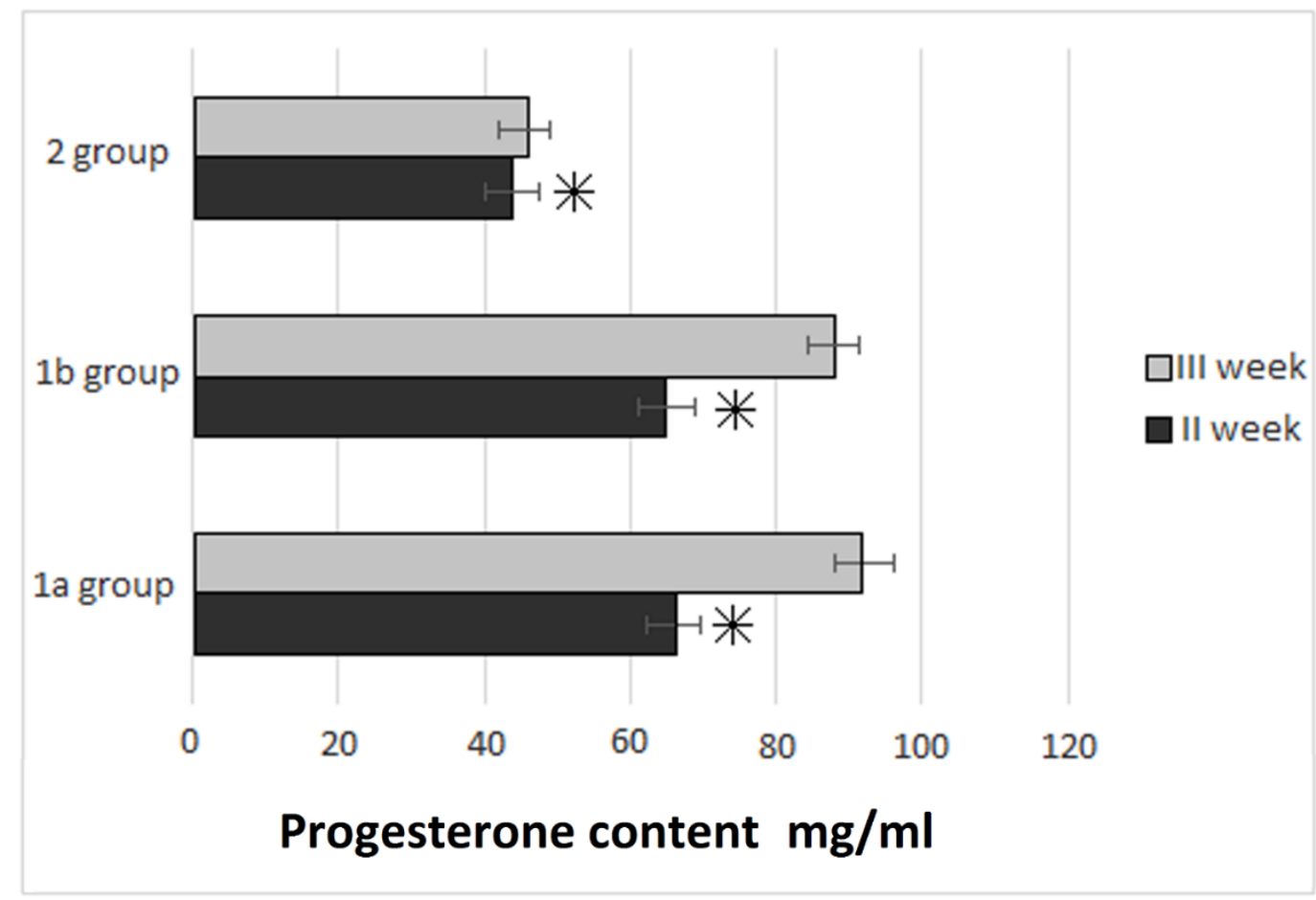

Fig. 5. Progesterone content in the rats' blood during physiological and complicated by CRU-PP pregnancy (group 1a - control non-operated rats (5 animals); group $1 \mathrm{~b}$ - control sham-operated rats (5 animals); group 2 - operated rats with CRU-PP (5 animals)). Bars marked with an asterisk - a significant difference between the values in the second and third trimester; $\mathrm{P} \leq 0.05$, according to Student's t-test (Mean干SD).

Mean Arterial Pressure. Chronic reductions in uterine perfusion pressure in pregnant rats resulted in significant increases in arterial pressure relative to normal pregnant rats. Fig. 6 illustrates the increase in arterial pressure of animals with CRU-PP (group 2) as compared to rats with physiological pregnancy (group 1a, 1b). MAP averaged $\approx 125,59 \mathrm{~mm} \mathrm{Hg}(\mathrm{P}<0.05)$ in the CRU-PP rats at day 19 of pregnancy, whereas in rats with physiological pregnancy at day 19 of gestation MAP averaged $\approx 97,89 \mathrm{~mm} \mathrm{Hg}$ (in intact nonpregnant rats (group 1i) MAP averages $\approx 104,25 \mathrm{~mm} \mathrm{Hg}$ ). 


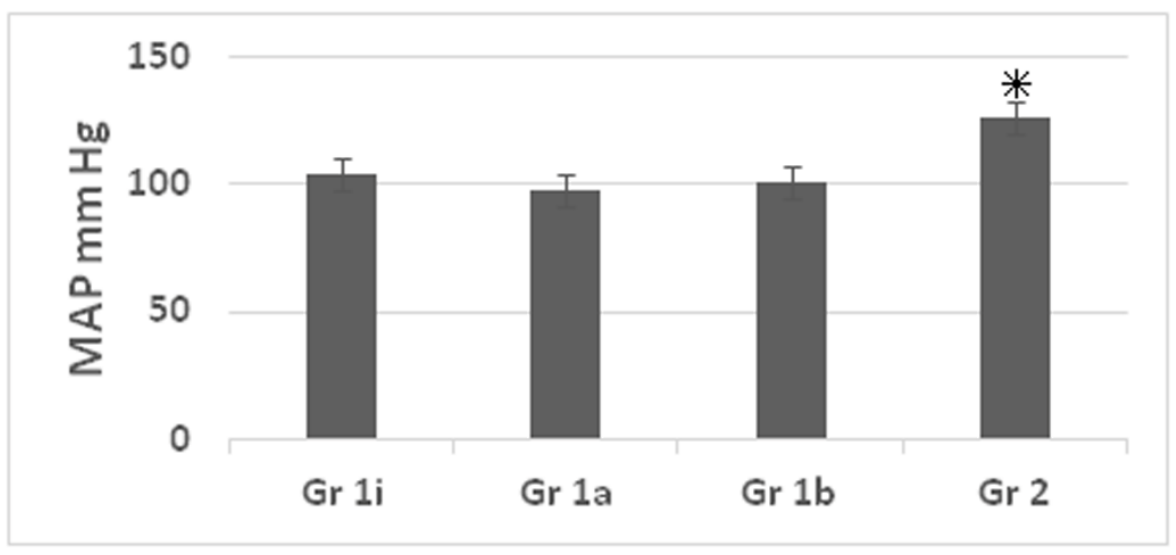

Fig. 6. Measurement of MAP in intact nonpregnant (group 1i), pregnant rats (with physiological pregnancy (group 1a), sham-operated pregnant rats (group 1b), and pregnant rats with CRU-PP (group 2)). Bars marked with an asterisk - a significant difference between the values of the MAP; P $\leq 0.05$, according to Student's ttest (Mean干SD).

\section{DISCUSSION}

The human placenta plays a central role in pregnancy, and the syncytiotrophoblast cells are the main components of the placenta that support the relationship between the mother and fetus, apart through the production of progesterone.

The syncytiotrophoblasts are formed by the fusion of underlying mononuclear cytotrophoblasts; upon differentiation into syncytiotrophoblast, their mitochondria appear to become highly specialized for steroidogenesis (Walter \& Miller, 2013; Holland et al, 2017; Martinez et al, 2015; Sanyal, 1978). The ability of rat's placenta to synthesize steroid hormones has been experimentally proved (it is noteworthy that the amount of progesterone synthesized by the placenta gradually increases with increasing gestational age) (Gordon et al, 1984; Arpad Csapo \& Wiest Walter, 1969; Sharashenidze et al, 2016). Progesterone, synthesized by the syncytiotrophoblasts from maternally derived cholesterol, plays a key role in the maintenance of pregnancy.

In our previous studies, impaired blood circulation and the development of hypoxia in the placenta of rats with experimental CRU-PP were found, which was manifested in a reduction of erythrocytes volume in fetal capillaries of the labyrinth layer, as well as decrease of proliferative activity of the blood vessels and obstructed visualization of endothelial cells (Sharashenidze A. et. al., 2016; Sharashenidze A. et.al., 2017). To establish the role of the hypoxic placenta in the pathogenesis of $\mathrm{PE}$, we investigated the regulatory mechanisms of steroidogenesis in the placenta during physiological pregnancy and pregnancy, complicated by a chronic reduction of the uteroplacental perfusion in rats.

The placental syncytiotrophoblasts contain at least two types of mitochondrial electron transport chains - one is responsible for mitochondrial respiration and synthesis of ATP, and the other - for steroidogenesis (more characteristic for these cells) (Sanyal, 1978). During steroidogenesis in the placenta, electrons from NADPH are transferred to the FAD-fragment of adrenodoxin reductase; then adrenodoxin reductase interacts with small iron-sulfur (FeS) protein, adrenodoxin, by the charge-charge attraction mechanism, which makes it possible to transfer electrons to FeS centers (Tuckey, 2005). Adrenodoxin interacts with binding-site of the redox-partner, mitochondrial cytochrome P450, transfers the electrons to its heme iron centers and provides catalysis of substrate binding to the cytochrome $\mathrm{P} 450$ ( $\mathrm{P} 450 \mathrm{scc}$ ). P450scc is specified by cleavage of the side chain of insoluble cholesterol to form soluble pregnenolone; this reaction is the ratelimiting step in the steroidogenesis. Pregnenolone can then converted into progesterone (Tuckey \& Headlam, 2002;--Tuckey, 2005). The activity of cytochrome P450scc in placental mitochondria, in turn, is limited to adrenodoxin reductase, which regulates the adrenodoxin pool in a fully reduced state through the electron delivery mechanism (Tuckey \& Headlam, 2002; Tuckey, 2005). Unlike other steroidogenic organs, the placenta does not have a short-term mechanism of modulation of steroid synthesis, and the intensity of steroidogenesis in the placenta is regulated by the redox state of the steroidogenic mitochondrial electron transport proteins. 
Intensification of steroidogenesis in the placenta during physiological pregnancy is usually accompanied by an increase in the intensity of electron flux through the adrenodoxin FeS centers - P450scc. In these reactions adrenodoxin plays the role of an electrons donor, and an increase in its content in the fully reduced state (manifested by an increase in the intensity of the EPR signal of the FeS centers of adrenodoxin paramagnetic in a reduced state) provides a flow of electrons to the heme iron of the mitochondrial P450scc, which binds cholesterol as a substrate (as a result, the intensity of the EPR signal of P450scc (paramagnetic in the oxidized (ferry), low spin state) decreases; P450scc converts it into soluble pregnenolone.

In the experimental model of CRU-PP an alterations in the EPR spectra of the hypoxic placenta was detected (a decrease in the intensity of the EPR signal of FeSadrenodoxin $\left(\mathrm{g}_{1}=1.94, \mathrm{~g}_{2}=2.02\right)$ and an increase in the intensity of the EPR signal of ferricytochrome P-450 ( $\mathrm{g}_{3}$ $=2.25)$ (Fig. 1, 2)), which may be associated with a decrease in the intensity of electron transport from adrenodoxin to P450scc in mitochondria (stage limiting the rate of steroidogenesis) and indicates a violation of placental steroidogenesis.

In the regulation of adequate perfusion in the placenta, there is no neuronal control and it is carried out mainly through physical factors and the circulation of the local tissue metabolites (Cartwright et al, 2002). Among these circulating factors, NO, characterized by pronounced vasodilation activity, is released (Myatt \& Cui, 2004; Myatt et al, 1996; Tropea et al, 2018). In the rat placenta (as well as in the human placenta) NO is synthesized by endothelial (eNOS) and inducible (iNOS) NO-synthases. eNOS is localized in syncytiotrophoblast cells inside the villi and the decidual trophoblast cells (Lyall F, 2005); iNOS was detected in the villous stroma of placenta (Baylis et al, 1999; Krause et al, 2011; Norris et al, 1999). It is generally accepted that the total synthesis of NO in the placenta increases during physiological pregnancy by eNOS from the end of the first trimester and can contribute to increasing of circulating NO content in peripheral blood (Norris et al, 1999; Motta-Mejia et al, 2017; Motta-Mejia et al, 2017). This coincides with the results of our study on an increase in the free NO content in the blood of pregnant rats (during an increase of gestation age) compared with its level in intact nonpregnant animals. However, the literature describing the production of NO in preeclampsia is very controversial (Possomato-Vieira \& Khalil, 2016; Matsubara et al, 2015; Darkwa et al, 2018; Laskowska et al, 2013; Khetsuriani et al, 2004; Hong-hai et al, 2011).

NO is a very reactive short-lived radical, rapidly converted into other more stable metabolites such as $\mathrm{NO}_{2}{ }^{-} / \mathrm{NO}_{3}{ }^{-}$, with very low bioactivity (Stamler et al, 1992; Mastronicola et al, 2003), or interacted with many other molecules such as proteins and metal ions (Sarti et al, 2003). S-nitrosylation of proteins is the mechanism of the redox-sensitive regulatory activity of $\mathrm{NO}$, which implies the reversible post-translational modification of proteins, signal transduction, regulation of the intensity of apoptosis and the function of trophoblasts in the placenta (Kingdom \& Kaufmann, 1997).

Due to the high affinity of NO to the heme and nonheme iron, free NO reversibly inhibits the mitochondrial heme and non-heme iron-containing electron-transport proteins (Stamler et al, 1992; Brown, 1995). These proteins react with NO by two alternative mechanisms leading to the formation of adducts (nitrosyl-adduct $\mathrm{Fe}_{2}-$ NO, or nitrite adduct) characterized by different recovery kinetics. The predominance of one mechanism over others depends on the flow rate of electrons through the mitochondrial electron transport chain. At the physiological electrons flow rate, $\mathrm{NO}$ at nanomolar concentrations effectively acts as a reversible regulator of the activity of the mitochondrial electron transport chain, and the formation of nitrites prevails (the nitrite adduct recovers much faster than the nitrosyl-one) (Brown, 1995). At high electrons flow rates formation of nitrosyl adduct may prevail over the nitrites formation (Giorgio et al, 1998); heme iron-containing proteins also lose their ability to form nitrites, which contributes to their nitrosylation. Also, only the hemenitrosyl adduct can release the still active NO into the surrounding environment. The level of nitrosyl heme adducts is an important indicator of the level of NO synthesis and its bioavailability in vivo (Khetsuriani et al, 2004; Redman \& Sargent, 2005; Khetsuriani et al, 2006; Tortladze et al, 2012).

Metabolic conditions lead to nitrosylation of mitochondrial proteins, reduce the level of free $\mathrm{NO}$ and can participate in the regulation of its content in the cell, but at the same time increase the risk of disruption of electron transport in the mitochondria - nitrosylation of non-heme FeS centers of proteins leads to their irreversible inhibition (Hong-hai et al, 2011). Thus, when the buffer/degrading ability of NO is disturbed and/or the synthesis/degradation rate of $\mathrm{NO}$ is not balanced, an excessive amount of free NO can become dangerous for the mitochondrial electron transport chain. This may be the case for CRP/UP accompanied by hypoxia in the placenta.

A significant decrease in the intensity of the EPR signal of spin-trapped free NO in the blood of rats with experimental CRU-PP (Fig. 4) and the appearance of EPR signals of nitrosyl complexes of heme (HbNO, $g_{c}=$ 2.01) and nonheme iron (FeSNO, $g=2.03$ ) in the EPR spectra of their placenta in the third week of pregnancy (Fig. 1, 2, 4) (group 1a, 1b) indicates intensive nitrosylation of heme iron of cytochrome P450 and FeS centers of the adrenodoxin in the steroidogenic mitochondrial chain of the placenta. In previous studies, 
high-intensity EPR signals from $\mathrm{HbNO}$ were detected by us in samples of the placenta in pregnant women with preeclampsia (Tortladze et al, 2012). Intensive incorporation of $\mathrm{NO}$ into the metal complexes of mitochondrial steroidogenic electron transport proteins in a hypoxic placenta, leads to a decrease in the free NO content in the body and reducing its biological activity, provides impairment of metabolism of placenta, irreversible inhibition of the mitochondrial steroidogenic electron transport on the stage of $\mathrm{FeS}$ centres of adrenodoxin - P450scc and decrease in the intensity of steroidogenesis, as evidenced by a sharp decrease in the content of progesterone in the rats' blood (Fig. 5).

\section{REFERENCES}

Arpad Csapo I., Wiest Walter G. (1969). An Examination of the Quantitative Relationship Between progesterone and the Maintenance of Pregnancy. Endocrinology. 85(735), 735746.

Baylis S.A., Strijbos P.J., Sandra A., Russell R.J., Rijhsinghani A., Charles I.G., Weiner C.P. (1999). Temporal expression of inducible nitric oxide synthase in mouse and human placenta. Mol. Hum. Reprod. 5(3), 277-86.

Beridze M., Sanikidze T., Shakarishvilil R., Intskirveli N., Bornstein N.M. (2011). Selected acute phase CSF factors in ischemic stroke: findings and prognostic value. $B M C$ neurology. 11(1), 41.

Brown G.C. (1995). Nitric oxide regulates mitochondrial respiration and cell functions by inhibiting cytochrome oxidase. FEBS Lett. 369, 136-139.

Cartwright J.E., Kenny L.C., Dash P.R., Crocker I.P., Aplin J.D., Baker P.N., Whitley G.S. (2002). Trophoblast Invasion of spiral arteries: a novel in vitro model. Placenta. 23(2-3), 232-5.

Chan S.W., Leathem J.H. (1975). Placental steroidogenesis in the rat: progesterone production by tissue of the basal zone. Endocrinology. 96(2), 298-303.

Crews J.K., Herrington J.N., Granger J.P., Khalil R.A. (2000). Decreased Endothelium-Dependent Vascular Relaxation During Reduction of Uterine Perfusion Pressure in Pregnant Rat. Hypertension. 35, 367-372.

Darkwa E. O., Djagbletey R., Essuman R., Sottie D., Dankwah G. B., Aryee G. (2018). Nitric Oxide and Pre-Eclampsia: A Comparative Study in Ghana. Open Access Maced J Med Sci. 6(6), 1023-1027.

Dash P.R., Cartwright J.E., Baker P.N., Johnstone A.P., Whitley G.S. (2003). Nitric oxide protects human extravillous trophoblast cells from apoptosis by a cyclic GMP-dependent mechanism and independently of caspase 3 nitrosylation. Exp Cell Res. 15 287(2), 314-24.

Dash P.R., Cartwright J.E., Whitley G.S. (2003). Nitric oxide inhibits polyamine-induced apoptosis in the human extravillous trophoblast cell line SGHPL- 4. Hum Reprod. 18(5), 59-68.

DutkaM., Pyka J., Plonka P.M. (2019). EPR Studies on Understanding the Physical Intricacy of HbNO Complexes.
Based on the results of our study, we can conclude that nitrosylation is an important redox-dependent mechanism by which NO regulates the activity of placental proteins involved in various metabolic pathways. During pathological pregnancy $(\mathrm{PE})$, the degree of placental hypoxia is an important factor determining the intensity of NO synthesis, its metabolism and the nitrosylation of mitochondrial steroidogenic electron transport chain proteins, which play an important role in the regulation of the intensity of steroidogenesis in the syncytiotrophoblasts and outcome of pregnancy.

In: Shukla EK (ed.), Electron Spin Resonance Spectroscopy in Medicine, Springer Nature Singapore Pte Ltd. 23-43.

Fink B., Dikakov S., Fink N. (2006). EPR techniques for detection of nitric oxide in vivo as an index of endothelial dysfunction. Pharmacological reports. 58, 8-15.

Gainutdinov Kh.L., Andrianov V.V., Gainutdinova T.Kh., Muranova L.N., Obynochny A.A., Timoshenko A.Kh., Shtark M.B., Epstein O.L., Yurtaeva S.V. (2009). Investigation of changes in NO content during Long-Term Sensitization in Edible Snail Using EPR-Spectroscopy: Effect of Antibodies to Calcium-Binding Protein S-100. Bulletin Experimental Biology and Medicine. 146(12), 617622.

Giorgio S., Linares E., Ischiropoulos H., Von Zuben F. J., Yamada A., Augusto O. (1998). In Vivo Formation of Electron Paramagnetic Resonance-Detectable Nitric Oxide and of Nitrotyrosine Is Not Impaired during Murine Leishmaniasis. Infect Immun. 66(2), 807-814.

George E.M., Granger J.P. (2012). Linking Placental Ischemia and Hypertension in Preeclampsia: Role of Endothelin 1. Hypertension. 60(2), 507-11.

Gordon J., Macdonald T., Mattf Dennis W. (1984). Adrenal and Placental Steroid Secretion during Pregnancy in the Rat. Endocrinology. 114(6), 2068-2073.

Granger J.P., LaMarca B.B., Cockrell K., Sedeek M., Balzi C., Chandler D., Bennett W. (2006). Reduced uterine perfusion pressure (RUPP) model for studying cardiovascular-renal dysfunction in response to placental ischemia. Methods. Mol.Med. 122, 383-92.

Holland O., Nitert M. Dekker, Gallo L.A., VejzovicM., Fisher J.J., Perkins A.V. (2017). Review: Placental mitochondrial function and structure in gestational disorders. Placenta. 54, 2-9.

Hong-hai Z., Wang Y. , Chen D. (2011). Analysis of NitrosoProteomes in Normotensive and Severe Preeclamptic Human Placentas. Biol Reprod. 84(5), 966-997.

Jakubowska M., Michalczyk-Wetula D., Pyka J, Susz A., Urbanska K., Płonka B.K., Kuleta Łacki P., KrzykawskaSerda M., Fiedor L., Płonka P. (2013). Nitrosylhemoglobin in hotodynamically stressed human tumors growing in nude mice. Nitric Oxide 35, 79-881. 
Khetsuriani T., Sanikidze T., Khugashvili R. (2004). Alterations of oxidative metabolism at the pregnancy attended with pre-eclampsia. Annals of Biomedical Research and Education. 4(2), 81-82.

Khetsuriani T., Chabashvili N., Sanikidze T. (2006). Role of endothelin-1 and nitric oxide level in pathogenesis preeclampsia. Georgian medical news. 141, 17-21.

Kingdom J.C., Kaufmann P. (1997). Oxygen and placental villous development: origins of fetal hypoxia. Placenta. 18(8), 613-21.

Kirima K., Tsuchiya K., Sei H., Hasegawa T., Shikishima M., Motobayashi Y., Morita K., Yoshizumi M., Tamaki T. (2003). Calculation of systemic blood NO dynamics by EPR spectroscopy: $\mathrm{HbNO}$ as an endogenous index of NO. Am J Physiol Heart Circ Physiol. 285, 589-96.

Klimek J., Woźniak M., Szymańska G., Zelewski L. (1998). Inhibitory effect of free radicals derived from organic hydroperoxide on progesterone synthesis in human term placental mitochondria. Free radical biology and medicine. 24(7-8), 1168-75.

Kozlov A.V., Szalay L., Umar F., Fink B., Kropik K., Nohl H., Redl H., Bahrami S. (2003). EPR analysis reveals three tissues responding to endotoxin by increased formation of reactive oxygen and nitrogen species. Free Radic Biol Med. 15, 1555-1562.

Kozlov A.V., Bini A.,, Iannone A,, Zini I., Tomasi A. (1996). Electron Paramagnetic Resonance Characterization of Rat Neuronal Nitric Oxide Production ex vivo. Methods in Enzymology, 268, 229-236.

Krause B.J., Hanson M.A., Casanello P. (2011). Role of nitric oxide in placental vascular development and function. Placenta. 32(11), 797-805.

Laskowska M., Laskowska K., Terbosh M., Oleszczuk J. (2013). A comparison of maternal serum levels of endothelial nitric oxide synthase, asymmetric dimethylarginine, and homocysteine in normal and preeclamptic pregnancies. Med Sci Monit. 6(6), 430-437.

Lobysheva II., Biller P., Gallez B., Beauloye C., Balligand JL. (2013). Nitrosylated hemoglobin levels in human venous erythrocytes correlate with vascular endothelial function measured by digital reactive hyperemia. PLOS ONE, 8(10), e76457

Lyall F. (2005). Priming and remodeling of human placental bed spiral arteries during pregnancy - a review. Placenta. 26, 31-6.

Manandhar B.L., Chongstuvivatwong V., Geater A. (2013). Antenatal care and severe pre-eclampsia in Kathmandu syncytiotrophoblast valley. J Chitwan Med College. 3(6), 4347.

Martinez F., Olvera-Sanchez S., Esparza-Perusquia M., GomezChang E., Flores-Herrera O. (2015). Multiple functions of syncytiotrophoblast mitochondria. Steroids. 103, 11-22.

Mastronicola D., Genova M. L., Arese M., Barone M. C., Giuffre A., Bianchi C., Brunori M., Lenaz G., Sarti P. (2003). Control of respiration by nitric oxide in Keilin Hartree particles, mitochondria and SH-SY5Y neuroblastoma cells. Cell Mol. Life Sci. 60, $1752-1759$.

Matsubara K., Higaki T., Matsubara Y., Nawa A. (2015). Nitric Oxide and Reactive Oxygen Species in the Pathogenesis of Preeclampsia. Int J Mol Sci. 16(3), 4600-4614.

Motta-Mejia C., Kandzija N., Zhang W., Mhlomi V., Cerdeira A. S., Burdujan A., Tannetta D., Dragovic R., Sargent I.L.,
Redman C. W., Kishore U., Vatish M. (2017). Placental Vesicles Carry Active Endothelial Nitric Oxide Synthase and Their Activity is Reduced in Preeclampsia. Hypertension. 70(2), 372-381.

Myatt L., Cui X. (2004). Oxidative stress in the placenta, Histochem. Cell Biol. 122, 369-382.

Myatt L., Rosenfield R.B., Eis A.L., Brockman D.E., Grrec L., Lyall F. (1996). Nitrotyrosine residues in placenta. Evidence of peroxynitrite formation and action. Hypertension. 28, 488493.

Nonaka A., Manabe T., Asano N., Kyogoku T., Imanishi K., Tamura K., Tobe T., Sugiura Y., Makino K. (1989). Direct ESR measurement of free radicals in mouse pancreatic lesions. Int J Pancreatol. 5(2), 203-211.

Norris L.A., Higgins J.R., Darling M.R., Walshe J.J., Bonnar J. (1999). Nitric oxide in the uteroplacental, fetoplacental, and peripheral circulations in preeclampsia. Obstet Gynecol. 93, 958-963.

North R.A., McCowan L.M., Dekker G.A., Plonka, P.M., Chlopicki S., Plonka B.K., Jawien J., Gryglewski R.J. (1999). Endotoxaemia in rats: detection of nitrosyl-haemoglobin in blood and lung by EPR, Curr. Top. Biophys. 23, 47-53.

Plonka PM., Chlopicki S., Wisniewska M., Plonka BK. (2003). Kinetics of increased generation of $\mathrm{NO}$ in endotoxemic rats as measured by EPR. Acta Biochim Pol. 50(3), 807-13.

Podjarny E, Losonczy G, Baylis C. (2004). Animal models of preeclampsia. Semin Nephrol. 24(6), 596-606.

Poston L., Chan E.H., Stewart A.W., Black M.A., Taylor R.S., Walker J.J., Baker P.N., Kenny L.C. (2011). Clinical risk prediction for pre-eclampsia in nulliparous women: development of model in international prospective cohort. BMJ. 342. d1875.

Possomato-Vieira J. S., Khalil R. A. (2016). Mechanisms of Endothelial Dysfunction in Hypertensive Pregnancy and Preeclampsia. Adv Pharmacol. 77, 361-431.

Pulatova M.K., Richireva G.T., Kuroptieva Z.V, (1989). Electron paramagnetic resonace in molekular radiobiology. Electroatomizdat. 72-80.

Redman C.W., Sargent I.L. (2005). Latest Advances in Understanding Preeclampsia. Science. 308, 1592-2005.

Rice-Evans C., Diplock A.T. (1992). Techniques in free radical research (laboratory techniques in biochemistry and molecular biology). Elsevier Science.181-89.

Roberts J.M., Cooper D.W. (2001). Pathogenesis and genetics of pre-eclampsia. Lancet. 357, 5356.

Sanikidze T.V., Beridze M., Mitagvaria N., Bakhtadze S., Khan N. (2012). Neuroprotective treatment of cerebral infarction: an experimental study. International Journal of Neuroscience. 123(2), 104-113.

Sanikidze T., Chikvaidze E. (2016). Role of the Free Radicals in Mechanisms of Gallstone Formation: An EPR Study. Radiat Prot Dosimetry. 172(1-3):317-324.

Sanyal M.K. (1978). Secretion of progesterone during gestation in the rat. J Endocrinol. 79(2), 179-90.

SartiP., AreseM., BacchiA., BaroneM.C., ForteE., Mastronicola D., BrunoriM., GiuffrèA. (2003). Nitric oxide and mitochondrial complex IV. IUBMB Life. 55(10-11), 605-11.

Sengupta P. (2013).The Laboratory Rat: Relating Its Age With Human's. Int J Prev Med. 4(6), 624-630.

Sharashenidze A., Kikalishvili L., Turmanidze T., Sanikidze T. (2016). Morphological Changes in Rat Placenta in Different 
Periods of Pregnancy during the Simulated Pre-Eclampsia. Georgian medical news. 236, 97-101.

Simpson E. R., Miller D. A. (1978). Cholesterol Side-Chain Cleavage, Cytochrome P450, and Iron-Sulfur Protein in Human Placental Mitochondria. Archives of Biochemistry and Biophysics. 190 (2), 800-808.

Sladek S.M., Magness R.R., Conrad K.P. (1997). Nitric oxide and pregnancy. Am J Physiol. 272, R441 R463.

Stamler J.S., Simon D.I., Osborne J.A., Mullins M.E., Jaraki O., Michel T., Singel D.J., Loscalzo J. (1992). S-nitrosylation of proteins with nitric oxide: synthesis and characterization of biologically active compounds. Proc Natl Acad Sci US A. 89, 444-448.

Tortladze M., Kintraia N., Sanikidze T. (2012). The EPR study of nitric oxide in placenta during preeclampsia. Georgian Med News. 208-209, 55-59.

Tropea T., Wareing M., Greenwood S.L., Feelisch M., Sibley C.P., Cottrell E.C. (2018). Nitrite mediated vasorelaxation in human chorionic plate vessels is enhanced by hypoxia and dependent on the NO-sGC-cGMP pathway. Nitric Oxide. 80, 82-88.

Tuckey R. C., McKinley A. J., Madeleine J. Headlam. (2001). Oxidized adrenodoxin acts as a competitive inhibitor of cytochrome P450scc in mitochondria from the human placenta. Eur. J. Biochem. 268, 2338-2343.

Tuckey R.C., Headlam M.J. (2002). Placental cytochrome P450sce (CYP11A1): comparison of catalytic properties between conditions of limiting and saturating adrenodoxin reductase. J Steroid Biochem Mol Biol. 81(2), 153-8.

Tuckey R.C. (2005). Progesterone synthesis by the human placenta. Placenta. 26(4), 273-281.

Walker J.J. (2000). Pre-eclampsia. Lancet. 356 (9237), 1260 1265.

Wan J., Hu Z., Zeng K., Yin Y., Zhao M., Chen M., Chen Q. (2018). The reduction in circulating levels of estrogen and progesterone in women with preeclampsia. Pregnancy Hypertens. 11, 18-25.

Walter L. Miller. (2013). Steroid hormone synthesis in mitochondria. Molecular and Cellular Endocrinology. 379(1-2), 1-84.

Wang J., Yang J., Wu X., Mu Y., Li S., Cui K., Wang X., Zhao F. (2014). Predictive value of placenta derived RASSF1A sequence expression in maternal plasma for pre-eclampsia. Zhonghua Yi Xue Yi Chuan Xue Za Zhi. 31(1), 25-8.

Wu Q., Wu G., Li J.X. (2016). Effect of hypoxia on expression of placental trophoblast cells SATB1 and $\beta$-Catenin and its correlation with the pathogenesis of preeclampsia. Asian Pac J Trop Med. 9(6), 567-71.

Zhang H., Wang Y., Chen D. (2011). Analysis of NitrosoProteomes in Normotensive and Severe Preeclamptic Human Placentas. Biology of Reproduction. 84(5), 966-975. 